\title{
Incidence and Aetiology of Gallstones Formation after Laparoscopic Sleeve Gastrectomy (Retrospective Study)
}

\author{
A.AYousef ${ }^{1}$, A.M.Zedan ${ }^{2}$, M.E.Zayed ${ }^{1}$, and M.A.Elmoghazy ${ }^{1}$ \\ ${ }^{1}$ General Surgery Dept., Faculty of Medicine, Benha Univ., Benha, Egypt \\ ${ }^{2}$ Oncology Surgery Dept., Faculty of Medicine, Benha Univ., Benha, Egypt \\ E-Mail: mahmoudabual3bbas241@gmail.com
}

\begin{abstract}
Gallstones have been shown to develop in patients who experience rapid weight loss after dietary restriction and bariatric surgery . Data regarding gallstone development after Sleeve Gastrectomy, however, are limited. The aim of this study was to evaluate the incidence of gallstones formation after laparoscopic sleeve gastrectomy as a common complication to rapid weight loss during one year period after operation.40 patients had been followed up after undergoing bariatric surgery for assessment of gallstone formation and results found according to sex, age, DM, hyperlipidemia, type of surgery, relation to BMI, rate of weight loss, timing of gallstone formation. The mean age of patients was 37 . and 27 patients were females (67.5\%). Half of patients came with morbid obesity (BMI from 35 to 40), and the other half with extreme morbid obesity (BMI >40). After one year of Sleeve Gastrctomy operation 8 patients developed gallstones while 32 patient were free from gallstone formation. According to sex, 5 females developed gallstones After one year of Sleeve Gastrctomy operation, while 2 males came with the same diagnosis, $p=0.27$, According to age, 4 cases Less than $40 \mathrm{y}$. presented with gallstone formation After one year of Sleeve Gastrctomy operation, while 3 cases More than 40 y. came with gallstone, $\mathrm{p}=0.75$. Gallstones are a common complication after rapid weight loss from laparoscopic sleeve gastrectomy. Routine prophylactic cholecystectomy is recommended for weight reduction during laparoscopic sleeve gastrectomy.
\end{abstract}

Keywords: Gallstones, Laparoscopic, Sleeve, Gastrectomy.

\section{Introduction}

Obesity is a major health problem worldwide and has reached an epidemic proportion in the Western society. Evidence continues to accumulate that obesity is a major risk factor for many diseases and is associated with significant morbidity and mortality ,The most widely accepted measure of obesity is the body mass index (BMI). This number is calculated by dividing a patient's mass $(\mathrm{kg})$ by his or her height $(\mathrm{m} 2)$. A normal BMI is considered in the range of $18.5-24.9 \mathrm{~kg} / \mathrm{m} 2$. A BMI of $25-$ $29.9 \mathrm{~kg} / \mathrm{m} 2$ is considered overweight. A BMI of $30 \mathrm{~kg} / \mathrm{m} 2$ or greater is classified as obese; this classification is further subdivided into class I, II, or III obesity, Considering other factors (eg, total muscle mass, waist circumference) besides the BMI may be important. For example, an extremely muscular individual may have an elevated BMI without being considered overweight. Waist circumference has been shown to be an excellent indicator of abdominal fat mass. A circumference of greater than 88 $\mathrm{cm}$ (35 in.) in women or greater than $102 \mathrm{~cm}$ (40 in.) in men strongly correlates with an increased risk of obesityrelated disease [1].

Bariatric surgery is currently the only modality that provides a significant, sustained weight loss for morbidly obese patients, with resultant improvement in obesityrelated comorbidities. A prospective, controlled Swedish study involving 4047 obese patients, half of whom had undergone bariatric procedures, followed up over 14.7 years, found that compared to usual care, bariatric surgery was associated with a significantly reduced number of cardiovascular deaths and a lower incidence of cardiovascular events in obese adults [2].

Sleeve gastrectomy has been used as the first stage of a two-stage procedure for high-risk patients, but owing to

its simplicity and favorable outcomes, it is currently being offered as a standalone primary procedure. In the first decade of the 21 st century, many hundreds of sleeve gastrectomies were performed in the United States. On the basis of follow-up periods of 6 months to 3 years, patients were found to have lost $33-83 \%$ of their excess weight [3].

Compared with other bariatric procedures, sleeve gastrectomy is the more physiologic treatment because it does not involve malabsorption, abnormal tracts, blind tracts, or the placement of a foreign body. This procedure is widely performed laparoscopically [4].

Based on this meta-analysis, RYGB is more effective than SG in improving weight loss and short- and mid-term glycaemic and lipid metabolism control in patients with and without T2DM. Therefore, unless contraindicated, RYGB should be the first choice to treat patients with obesity and T2DM and/or dyslipidaemia [5].

Gallstone formation is common after LSG though incidence of complicated stones is small. This is despite not using gallstone-lowering prophylaxis. The low conversion rate also questions the relevance of surveillance screening, as most patients with new gallstones remain asymptomatic at least in the short-term follow-up, Gallstones are a common complication after rapid weight loss from SG. Our data suggest that gallstone formation during the weight loss period is not associated with amount or rate of weight loss both during the early or late postoperative period [6].

Study determines the incidence of and potential risk factors related to the development of gallstones following laparoscopic sleeve gastrectomy. The data of patients who underwent laparoscopic sleeve gastrectomy at a single center due to morbid obesity between January 2014 and 
December 2017 was retrospectively reviewed and analyzed. The patients were divided into two groups, as those with gallstones detected on ultrasound at 12 months and those without gallstones. Data of the two groups was compared. BMI did not differ significantly between patients with positive (+) and negative (-) ultrasound findings $(p>0.05)$. Aside from age, hypertension, and coronary artery disease, other preoperative parameters showed no significant association with the development of gallstones in USG (-) and USG (+) patients. The present study identified no significant relationship between a decrease in BMI following LSG and the postoperative development of gallstones. Preoperative hypertension and coronary artery disease were found to be significantly related to the development of gallstones after surgery. The authors suggest that patients with preexisting $\mathrm{CAD}$ and hypertension in the preoperative period must be followed-up with ultrasound more meticulously [7].

Traditional risk factors for gallstone formation in the general population are not predictive of symptomatic gallstone formation after bariatric surgery. Weight loss of more than $25 \%$ of original weight was the only postoperative factor that can help selecting patients for postoperative ultrasound surveillance and subsequent cholecystectomy once gallstones were identified [8].

The aim of this study was to evaluate the incidence of gallstones formation after laparoscopic sleeve gastrectomy as a common complication to rapid weight loss during one year period after operation.

\section{Patients and methods}

Our prospective study was conducted in General Surgery Department of Benha University Hospital after an approval from the research ethics committee in Benha Faculty of Medicine and all patients signed arabic informed consents that they were involved in this study. A total of 100 patients with a history of recently performed sleeve gastrectomy was recruited to our study .

\subsection{Inclusion criteria}

1- Morbidly obese patients with no history of performing cholecystectomy.

2- Morbidly obese patients without evidence of GB stones.

Table (1) General characteristic.

\subsection{Exclusion criteria}

1- Morbidly obese patients with history of cholecystectomy.

2- Morbidly obese patients with evidence of GB stones.

3 - Type 1 and type 2 diabetes mellitus,

4- A history of cardiovascular disease (CVD),

5- Secondary cause of obesity

All patients meeting the inclusion criteria was subjected to our study after proper history taking (Name ,Age, Sex, Address, Body mass index is 35 or higher ) full clinical examination required preoperative investigations Laboratory as CBC, lipid profile, liver \& kidney functions, Imaging as pelvi-abdominal US.

Patients had been informed about the techniques and the complications with a written consent.

When Body mass index (BMI) was 40 or higher patient was cosidered (extreme morbid obesity) while (BMI) from 35 to 39.9 before surgery was consedered (morbid obesity)

Transabdominal ultrasound (US) was performed in all patients preoperatively to rule out gallstones or sludge. According to the protocol, patients with positive findings on ultrasound were counselled for concomitant laparoscopic cholecystectomy.

\subsection{Statistical analysis}

The collected data was tabulated and presented in suitable figures. Quantitative data was summarized using mean and standard deviation, while, qualitative data by using frequency and percentage. Data was analyzed by the aid of software package of SPSS using suitable statistical tests. The accepted level of significance in this work was 0.05 ( $\mathrm{P}<0.05$ was considered significant).

\section{Results}

In this study, 40 patients had been followed up after undergoing bariatric surgery for assessment of gallstone formation and results found according to sex, age, DM, hyperlipidemia, type of surgery, relation to BMI, rate of weight loss, timing of gallstone formation. The mean age of patients was 37 years, and 27 patients were females, Table (1)

\begin{tabular}{llcc}
\hline Age (years) & \multicolumn{2}{c}{ Mean \pm SD } & $31 \pm 8$ \\
\hline Gender & Males & $\mathrm{n}(\%)$ & $13(32.5 \%)$ \\
& Females & $\mathrm{n}(\%)$ & $27(67.5 \%)$ \\
BMI & Morbid obesity & $\mathrm{n}(\%)$ & $20(50.0)$ \\
& extreme morbid obesity & $\mathrm{n}(\%)$ & $20(50.0)$ \\
Total & & & 40 \\
\hline
\end{tabular}

$* \mathrm{BMI}=$ Body mass index

After one year of Sleeve Gastrctomy operation 8 patients developed gallstones while 32 patient were free from gallstone formation Fig (1) 


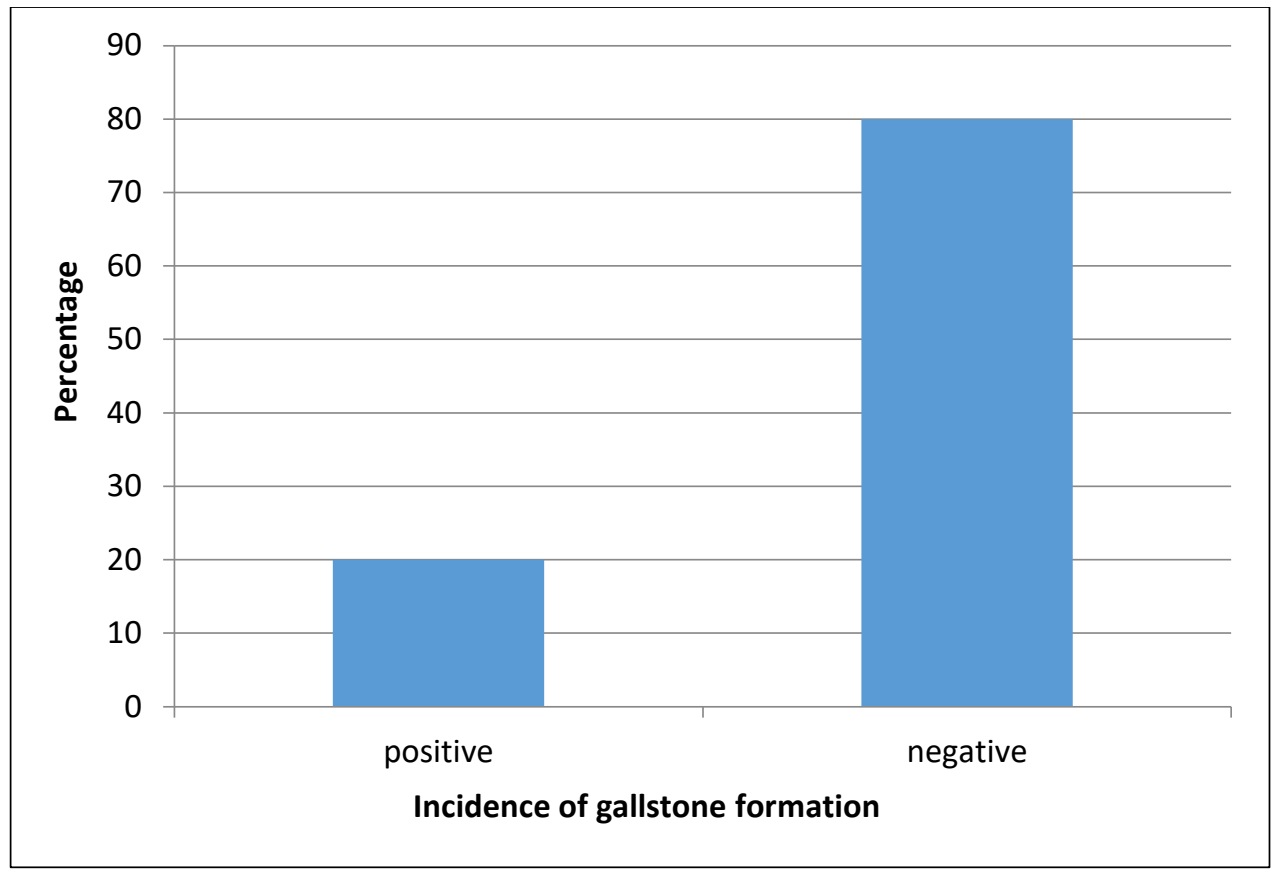

Fig (1) Incidence of gallstone formation

According to sex, 5 females developed gallstones After one year of Sleeve Gastrctomy operation, while 2 males came with the same diagnosis. The P-value of this study was 0.27 which is insignificant as shown in Table (2).

Table (2) Incidence of gallstone formation after bariatric surgery in relation to sex.

\begin{tabular}{lcccc}
\hline Sex & Males & Females & $\begin{array}{c}\text { Chi } \\
\text { square }\end{array}$ & p-value \\
\hline +ve gallstone formation(Yes) & 2 & 5 & & \\
-ve gallstone formation(No) & 11 & 22 & 1.2191 & 0.269544 \\
Total & 13 & 27 & & \\
\hline
\end{tabular}

$*(\mathrm{P}<0.05)$

According to age, 4 cases Less than $40 \mathrm{y}$. presented with gallstone formation After one year of Sleeve Gastrctomy operation, while 3 cases More than $40 \mathrm{y}$. came with gallstone. The P-value of this study was 0.75 which is insignificant as shown in Table (3).

Table (3) Incidence of gallstone formation after bariatric surgery in relation to age.

\begin{tabular}{|c|c|c|c|c|}
\hline Age & Less than $40 \mathrm{y}$. & More than $40 \mathrm{y}$. & $\begin{array}{c}\text { Chi } \\
\text { square }\end{array}$ & p-value \\
\hline +ve gallstone formation(Yes) & 4 & 3 & & \\
\hline -ve gallstone formation(No) & 21 & 12 & 0.1039 & 0.747203 \\
\hline Total & 25 & 15 & & \\
\hline
\end{tabular}

According to BMI, one case of previously morbid obesity developed gallstones After one year of Sleeve Gastrctomy operation, while 6 cases with preoperative extreme morbid obesity came with the same diagnosis . The P-value of this study was 0.037 which is significant as shown in Fig (2). 


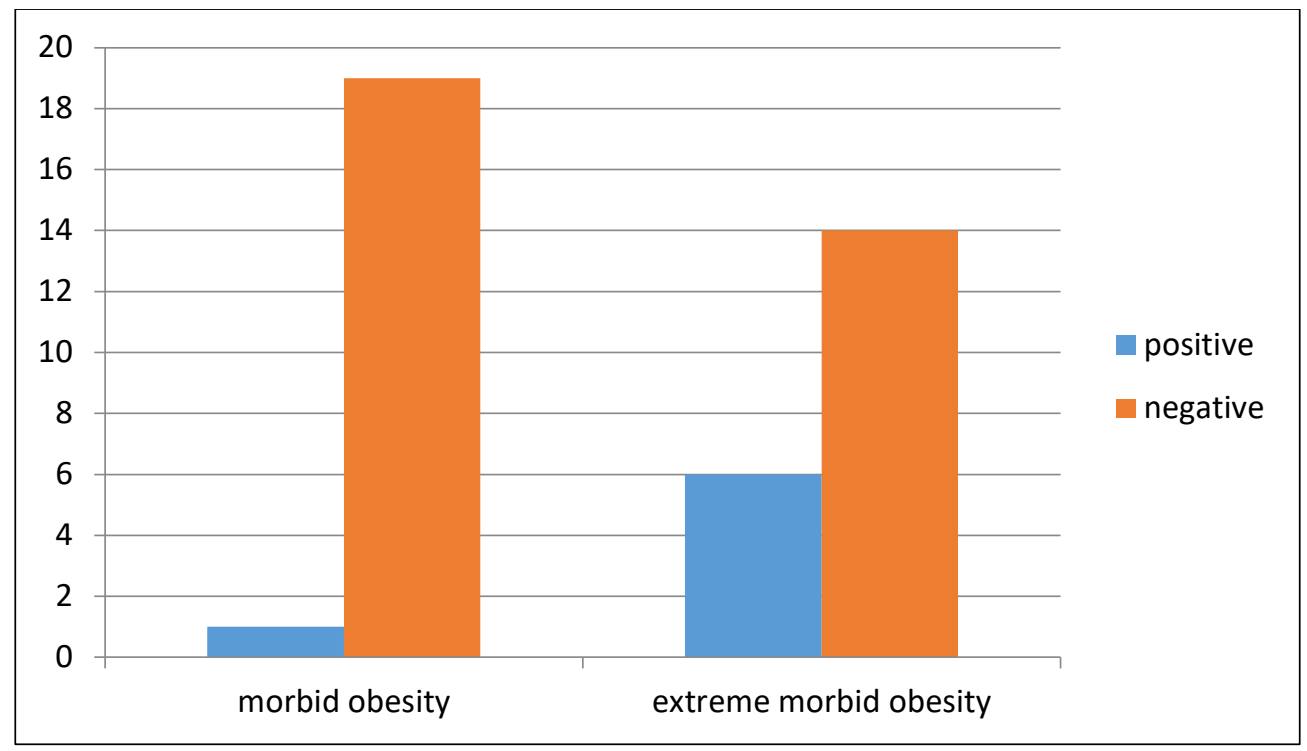

Fig (2) Diagram showing the incidence of gallstone formation after bariatric surgery according to BMI.

According to Rate of weight loss, 6 cases who loosed More than $25 \%$ of their weight after one year of Sleeve Gastrctomy operation developed gallstones, while 1 case from whom loosed less than $25 \%$ of their weight came with the same diagnosis. The P-value of this study was 0.025 which is significant as shown in Fig (3).

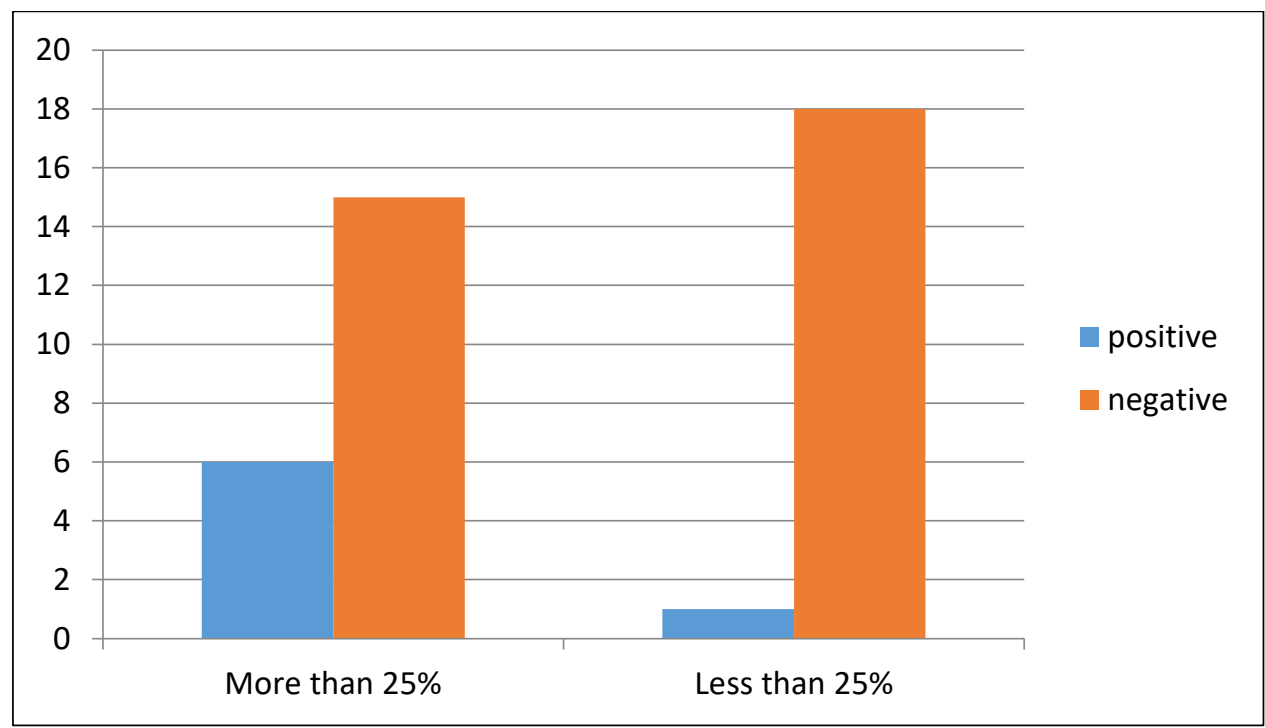

Fig (3) Diagram showing the incidence of gallstone formation after bariatric surgery according to rate of weight loss.

\section{Discussion}

The aim of this study was to evaluate the incidence of gallstones formation after laparoscopic sleeve gastrectomy as a common complication to rapid weight loss during one year period after operation.

In this study, 40 patients had been followed up after undergoing bariatric surgery for assessment of gallstone formation and results found according to sex, age, DM, hyperlipidemia, type of surgery, relation to $\mathrm{BMI}$, rate of weight loss, timing of gallstone formation.

In the present study, There mean age of patients was 37 years, and 27 patients were females $(67.5 \%)$. Half of patients came with morbid obesity (BMI from 35 to 40), and the other half with extreme morbid obesity

(BMI $>40$ ).

In the same with Sioka, et.al., [9] study The median age was 40 years (range 18-62) and the median BMI was 46.1 (range 35-61). Patients in this study were predominantly female (79\%). In [6] study the mean age was 42.9 years(range, $17-81$ years) for group A, and 370 $(74.6 \%)$ of thepatients were women

In the present study, After one year of Sleeve Gastrctomy operation 7 patients developed gallstones (17.5\%) . In V. K. M. Li et al., [6] study postoperative gallbladder disease was present in $(25.3 \%)$ of patients. In W. Manatsathit et al., [10] study the incidence of 
symptomatic gallstones was 22.9\% (22/96) including 13 patients biliary colic, 5 patients with cholecystitis, 3 patients with obstructive jaundice, and one patient with pancreatitis.

In the present study, According to sex, 5 females developed gallstones After one year of Sleeve Gastrctomy operation, while 2 males came with the same diagnosis . The P-value of this study was 0.27 which is insignificant . According to age, 4 cases Less than 40 y. presented with gallstone formation After one year of Sleeve Gastrctomy operation, while 3 cases More than $40 \mathrm{y}$. came with gallstone. The P-value of this study was 0.75 which is insignificant

In W. Manatsathit et al., [10] study There were no significant differences between the two groups by gender, race or mean age, initial BMI or initial weight. Lipid profile values were also similar in both groups.

In the opposite side M. J. Stampfer et al., [11] reported that risk factors for gallstone formation after bariatric surgery such as female gender and increasing age were well known to surgeons..

In the present study, According to BMI, one case of previously morbid obesity developed gallstones After one year of Sleeve Gastrctomy operation, while 6 cases with preoperative extreme morbid obesity came with the same diagnosis. The P-value of this study was 0.037 which is significant

The risk of gallstone formation increased 8 -fold in patients with BMI more than $40 \mathrm{~kg} / \mathrm{m} 2$, it also increased 5-fold in patients who underwent bariatric surgery compared with normal population [12].

The results of B. T. Grover et al., [13] that said gallstones formation increased 8-fold in patients with BMI more than $40 \mathrm{~kg} / \mathrm{m} 2$.

In the present study, According to Rate of weight loss, 6 cases who loosed More than $25 \%$ of their weight after one year of Sleeve Gastrctomy operation developed gallstones, while 1 case from whom loosed less than $25 \%$ of their weight came with the same diagnosis. The Pvalue of this study was 0.025 which is significant.

The postoperative factor of weight loss of more than $25 \%$ of original weight was found to be associated with gallstone formation [6].

Also more than a $24 \%$ loss of original body weight was found to be a significant risk factor for gallstone formation [14].

In our group of patients, the postoperative factor of weight loss of more than $25 \%$ of original weight was found to be associated with symptomatic gallstone formation, those patients with weight loss of more than this were likely to become symptomatic even if they were not symptomatic at the time of gallstone detection. These patients had to do cholecystectomy once gallstones were identified despite being asymptomatic.

The prevalence of cholesterol gallstones was high among obese persons. Weight loss further increased the risk of gallstones: the prevalence of new gallstones reached more than $30 \%$ within 12-18 months after gastric bypass surgery. The increased occurrence of stones was commonly because of supersaturation of bile with cholesterol, due to an increased synthesis by the liver and secretion into bile [14].

Identification of predictive factors for gallstone formation after weight reduction surgery might be important in selecting patients for certain prophylactic interventions as regular ultrasound surveillance for gallstones [15].

Several studies also further attempted to identify risk factors associated with gallstone formation after bariatric procedures and consistently demonstrated that, unlike general population, conventional risk factors for gallstone development such as age, gender, and diabetes were not associated with gallstone formation during rapid weight loss after bariatric surgery, in fact, it was believed that more rapid and higher amount of weight loss contributed to higher incidence of gallstones [6].

\section{Conclusion}

Gallstones are a common complication after rapid weight loss from laparoscopic sleeve gastrectomy. Routine prophylactic cholecystectomy is recommended for weight reduction during laparoscopic sleeve gastrectomy.

\section{References}

[1] S. Doll, F. Paccaud, P. al Bovet, M. Burnier, and V. Wietlisbach, "Body mass index, abdominal adiposity and blood pressure: consistency of their association across developing and developed countries," Int. J. Obes, Vol. 26, (1), PP. 48-57, 2002.

[2] S. Romeo, "Cardiovascular events after bariatric surgery in obese subjects with type 2 diabetes," Diabetes Care, Vol. 35(12), PP. 2613-2617, 2012.

[3] G. P. Kohn, J. A. Galanko, D. W. Overby, "Recent trends in bariatric surgery case volume in the United States," Surgery, Vol. 146(2), PP. 375-380, 2009.

[4] H. Christellea, H. Thierrya, R. D. Aristotleab, "Comparison of metabolic outcomes in patients undergoing laparoscopic roux-en-Y gastric bypass versus sleeve gastrectomy -a systematic review and meta-analysis of randomised controlled trials," Swiss Med Wkly, Vol. 148, PP. w14633, 2018.

[5] S. Ikramuddin, "Lifestyle intervention and medical management with vs without Roux-en-Y gastric bypass and control of hemoglobin A1c, LDL cholesterol, and systolic blood pressure at 5 years in the diabetes surgery study," Jama, Vol. 319(3), PP. 266-278, 2018.

[6] V. K. M. Li, N. Pulido, P. Fajnwaks, "Predictors of gallstone formation after bariatric surgery: a multivariate analysis of risk factors comparing gastric bypass, gastric banding, and sleeve gastrectomy," Surg. Endosc, Vol. 23(7), PP. 16401644, 2009.

[7] S. Özdaş , H. Bozkurt, "Factors Affecting the Development of Gallstones Following Laparoscopic Sleeve Gastrectomy," Obes. Surg., Vol. 29(10), PP. 3174-3178, 2019.

[8] L. D. MacLean, B. M. Rhode, C. W. Nohr, "Late 
outcome of isolated gastric bypass," Ann. Surg., Vol. 231, (4), PP. 524, 2000.

[9] E. Sioka, "Complicated gallstones after laparoscopic sleeve gastrectomy," J. Obes, Vol. 2014, (2), PP. 65-78, 2014.

[10] W. Manatsathit, P. Leelasinjaroen, H. Al-Hamid, "The incidence of cholelithiasis after sleeve gastrectomy and its association with weight loss: A two-centre retrospective cohort study," Int. J. Surg, Vol. 30, PP. 13-18, 2016.

[11] M. J. Stampfer, K. M. Maclure, G. A. Colditz, "Risk of symptomatic gallstones in women with severe obesity," Am. J. Clin. Nutr, Vol. 55(3), PP. 652-658, 1992.

[12] E. Jonas, R. Marsk, F. Rasmussen, "Incidence of postoperative gallstone disease after antiobesity surgery: population-based study from Sweden," Surg. Obes. Relat. Dis, Vol. 6(1), PP. 54-58, 2010.

[13] B. T. Grover, S. N. Kothari, "Biliary issues in the bariatric population," Surg. Clin., Vol. 94(2), PP. 413-425, 2014.

[14] S. Erlinger, "Gallstones in obesity and weight loss.," Eur. J. Gastroenterol. Hepatol, Vol. 12(12), PP. 1347-1352, 2000.

[15] D. J. Scott, L. Villegas, T. L. Sims, "Intraoperative ultrasound and prophylactic ursodiol for gallstone prevention following laparoscopic gastric bypass," Surg. Endosc. Other Interv. Tech, Vol. 17(11), PP. 1796-1802, 2003. 